\title{
Late acceptance hill climbing algorithm for solving Patient facing problems in Hospitals
}

\author{
Irfan Majeed ${ }^{1}$, Dr. Mansoor Alam², M. Mustaneer Noor ${ }^{3}$, Rizwan Ahmed ${ }^{4}$, M. Humayoun ${ }^{5}$, Javeria \\ Iftikhar $^{6}$
}

\begin{abstract}
The current study is to evaluate the patient facing problems by applying Late Acceptance Hill Climbing Algorithm (LAHC) in hospital settings. The recent proposed procedure of $\mathrm{LAHC}$ is based on metaheuristic algorithm which is linked with one-point clarification method. Patient's satisfaction regarding the performance of the hospital is a composite mechanism. The optimization procedure is connected with NP-hard problems which is practically related to the problems faced by patients. These problems are concerned with assigning the group of patients visiting the hospital for receiving healthcare services. The common issues faced by patients include communication gap, response time to attend patients, early symptomatic relief, getting proper advice for dosage and usage of medicines, clean hospital environment. Moreover, patient education and guidance before discharge from hospital is also the missing element. The suggested algorithm of LAHC to PFP is developed and it has two phases: the first phase includes providing the initial feasible solution using communication-oriented methodology. The second phase uses three neighborhood framework which are implanted inside the segment of PFP based on LAHC to additionally upgrade the underlying feasible solution of the introductory phase.
\end{abstract}

1,2,4,5,6Institute of Business Management (IoBM),

Karachi-75190, Pakistan.

1irfan.majeed001@gmail.com

${ }^{2}$ Malaysian Institute of Information Technology

(MIIT), Malaysia

${ }^{2}$ University of Kuala Lumpur

${ }^{3}$ University of Karachi, Pakistan

${ }^{1,3}$ Indus Pharma (Pvt.) Ltd.

${ }^{2}$ mansoor@unikl.edu.my
Furthermore, the LAHC strategy is assessed utilizing the standard benchmark dataset. The exploratory outcomes determined that the professional presented strategy is a successful procedure for handling the PFP issues. The study revealed that the method applied is far better compare to the non-contemporary methods adopted to fulfill patient needs.

Keywords: Patient facing problems, Late acceptance hill climbing, Metaheuristic

\section{INTRODUCTION}

It is observed that in both developed and developing countries the standard of healthcare facilities doesn't meet to the general population expectation. A large number of the population in many countries particularly in rural area, not aware about the latest healthcare facilities. On the other hand people living in urban areas have opportunity to get benefit from the latest healthcare developments. Health is the basic requirement for living a happy life. This is the dilemma in our society the underprivileged population is unable to receive basic healthcare facilities and which make their life miserable. If any household member gets any critical illness the whole family suffers due to unavailability of proper healthcare services. In other words, there is an increased frustration among people living in developing countries for not receiving basic health facilities. ${ }^{1}$

Patient come in hospital with a fixed mind set to receive better healthcare services. One of the most common concern is to satisfy the need of patient faced problems (PFP) in hospitals at the time of visit for consultation and admission. The activities of PFP may be considered because number of patients go to different hospitals and faced unavailability of adequate resources in hospitals. For instance, absence of welltrained doctors, paramedical staff, clean rooms, beds, 
janitors and recovery time are predetermined class of constraints. The frequent imperatives in PFP may be categorized into hard and soft constraints. Due to nature of PFP, rendering feasible solution that fascinate the entire soft constraints is virtually complicated. ${ }^{2}$ Moreover, several formulation have been proposed from the literature. ${ }^{3}$ The algorithmic procedures linked through the investigators for PFP are specifically accessed, heuristics-based and metaheuristic-based techniques. ${ }^{4}$ Different studies suggest precise strategies for the PFP. ${ }^{5-6}$ The concept based on the LAHC has a late acceptance approach that is used to anticipate the algorithmic program, which is bound in a local minimum search that usually similar to several greedy search algorithms. Due to its integrity, it's been effectively practiced, adopted and revised as per the researchers within the domain of computing and research to address various improvement issues like leveling two-sided assembly lines, ${ }^{7}$ exam time tabling, ${ }^{8}$ lock scheduling, ${ }^{9}$ linear shipping fleet repositioning, ${ }^{10}$ and travelling salesman problem. ${ }^{11}$ Therefore, the aim of our research is to evaluate the functioning of LAHC algorithm for figuring out the PFP in hospitals.

\section{PATIENT FACED PROBLEMS IN HOSPITALS}

At the time of admission or discharge from hospitals common issues faced by patients are communication gap between doctor and nurses, delayed response from paramedics, symptoms aggravation, poor pain management, inadequate information about usage of medicine, rooms and bathroom cleaning and lack of instruction. Although, these are associated with patient's condition and time-interval during stay in hospital. For instance, in pursuit of diagnosis and treatment, requests for medical facilities, age, stratification, gender, room reference, confirmation date of admission, patient's length of stay in hospital, availability of medicine and laboratory tests. To facilitate patient's medical institutions should also install medical apparatus in room, electric board for cell phone charging, extra bed and the area of the bed in the ward. It can be suggested that as per disease specification and target specialties the hospital must ensure the availability of medical equipment in patients' rooms and wards to ascertain right diagnosis and treatment of disease. However, during hospital stay patients should receive proper and timely advice regarding self-care. Patients must receive timely medical attention. There should be a standard process for confirmation span regarding hospital stay of admitted patients.

The detailing of the PFP issue is contemplated in this exploration that comprise six hard constraints (i.e., $\mathrm{H}_{1}$
- $\mathrm{H}_{6}$ as considered in Table 1) that should be fulfilled so as to accomplish possible outcomes.

\section{PROBLEM FORMULATIONS}

The Patient facing problems (PFP) of allocating a set of $\mathrm{n}$ patients visiting $\beta=\left\{p_{1}, p_{2}, \ldots \ldots, p_{n}\right\}$ to $\mathrm{c}$ set of (doctors and nurses well communicate with patients) $\beta=\left\{d_{1}, d_{2}, \ldots, d_{c}\right.$ ), q set of (quick response time to attend patients) $\beta=\left\{h_{1}, h_{2}, \ldots ., h_{q}\right\}$ in a $\mathrm{p}$ set of (patients receive early symptomatic relief) $\beta=$ $\left\{c_{1}, c_{2}, \ldots, c_{p}\right\}, \mathrm{m}$ set of (patients getting proper advice for dosage and usage of medicines) $\mid \beta=$ $\left\{e_{1}, e_{2}, \ldots, e_{m}\right\}, \mathrm{r}$ set of (clean hospital environment especially rooms and bathrooms) $\beta=$ $\left\{b_{1}, b_{2}, \ldots, b_{r}\right\}$ and $\mathrm{h}$ set (patient education and guidance before discharge from hospital) $\beta=$ $\left\{i_{1}, i_{2}, \ldots, i_{h}\right\}$. The PFP solution is presented by the vector $w=\left(w_{1}, w_{2}, \ldots, w_{n}\right)$ of the patients, where the scores of $w_{i}$ is a map of communicate with patient's $\mathrm{d}_{\mathrm{c}}$ and quick response time $h q$ for the patients $\mathrm{i}$ :

\section{DEFINITIONS}

Definitions of the constraints (soft and hard) in PFP:

\section{IV. i: Definitions}

The perspective of $i$ is executable for the patients $w_{i}$ be allocated to the outcome $\mathrm{w}$ in the event satisfying the requirements as described below,

Formulation of the first hard constraint may be defined as

$$
\forall c \in d, \forall h \in q, \sum_{p \epsilon d} \varrho_{p, d, h} \leq 1
$$

The hard constraints of $1^{\text {st }}, 2^{\text {nd }}, 3^{\text {rd }}$ and $4^{\text {th }}$ can be expressed as

$$
\forall c \in d, \forall h \in P S p, \sum_{c \in d} \varrho_{p, d, h}=1
$$

The hard constraint of $5^{\text {th }}$ and $6^{\text {th }}$ can be presented as:

$\forall b \in r, \forall i \in h, \Sigma_{p \in r} \Sigma_{p \in h} W p, r, h \leq C d$

\section{IV. ii: Definition 2: Formulation of Soft constraints.}

The information of the total consequences (GP) is assumed as:

$G P=\Sigma_{-} b \epsilon h \Sigma_{-} q \epsilon h \min (f b, q, z b, q)$ 
Table 1: Hard and Soft constraints of PFP problems

\section{Hard constraints}

$\mathrm{H}_{1}$ : Doctors and nurses communicate well with patients.

$\mathrm{H}_{2}$ : Quick response time to attend patients.

$\mathrm{H}_{3}$ : Patients receive early symptomatic relief.

$\mathrm{H}_{4}$ : Patients getting proper advice for dosage and usage of medicines.

$\mathrm{H}_{5}$ : Clean hospital environment especially rooms and bathrooms

$\mathrm{H}_{6}$ : Patient education and guidance before discharge from hospital.

\section{Soft constraints}

$\mathrm{S}_{1}$ : Admission and discharge process should be convenient

$\mathrm{S}_{2}$ : Availability of medicines and laboratory tests should be conducted in hospital premises

$\mathrm{S}_{3}$ : Patients should be satisfying with the equipment of the ward.

$\mathrm{S}_{4}$ : Hygienic food should be provided

$\mathrm{S}_{5}$ : Transportation services should be available within 24 hours

Table 2: Satisfaction scores of PFP

\begin{tabular}{ccccc}
\hline Cases & Mean & Standard deviation & Minimum & Maximum \\
\hline Case 1 & 70.55 & 6.864 & 57 & 79 \\
Case 2 & 49.45 & 4.083 & 44 & 57 \\
Case 3 & 57.64 & 3.042 & 53 & 64 \\
Case 4 & 53.73 & 4.338 & 45 & 61 \\
Case 5 & 63.36 & 4.717 & 56 & 73 \\
Case 6 & 78.55 & 2.423 & 75 & 82 \\
\hline
\end{tabular}

Table 3: Patient's satisfaction score with respect to grading

\begin{tabular}{|c|c|c|c|}
\hline Outcomes & Poor & Mixed & Better \\
\hline Doctors and nurses communicate well with patients. & $66.8(7.6 \%)$ & $68.4(6.7 \%)$ & $70.5(6.0 \%)$ \\
\hline Quick response time to attend patients. & $53.5(8.8 \%)$ & $54.8(7.3 \%)$ & $56.4(6.6 \%)$ \\
\hline Patients receive early symptomatic relief. & $62.8(6.5 \%)$ & $64.0(5.7 \%)$ & $65.9(4.8)$ \\
\hline Patients getting proper advice for dosage and usage of medicines. & $51.6(6.5 \%)$ & $53.3(5.9 \%)$ & $55.8(5.3 \%)$ \\
\hline Clean hospital environment especially rooms and bathrooms & $61.7(7.2 \%)$ & $63.2(6.7 \%)$ & $65.5(6.7 \%)$ \\
\hline Patient education and guidance before discharge from hospital. & $53.5(8.8 \%)$ & $54.8(7.3 \%)$ & $56.4(6.6 \%)$ \\
\hline
\end{tabular}

Source: HCAPHS; distribution outcomes express as Mean and percentages.

Table 4: Neighborhood Structure on LAHC for PFP

\begin{tabular}{l|cccccc}
\hline Searching of Neighborhood & $\mathbf{H}_{\mathbf{1}}$ & $\mathbf{H}_{\mathbf{2}}$ & $\mathbf{H}_{\mathbf{3}}$ & $\mathbf{H}_{\mathbf{4}}$ & $\mathbf{H}_{\mathbf{5}}$ & $\mathbf{H}_{\mathbf{6}}$ \\
\hline Entity Relocate & $\sqrt{ }$ & $\mathrm{X}$ & $\sqrt{ }$ & $\sqrt{ }$ & $\mathrm{X}$ & $\mathrm{X}$ \\
Entity Swap & $\sqrt{ }$ & $\mathrm{X}$ & $\mathrm{X}$ & $\mathrm{X}$ & $\sqrt{ }$ & $\sqrt{ }$ \\
Interchange & $\sqrt{ }$ & $\sqrt{ }$ & $\sqrt{ }$ & $\mathrm{X}$ & $\mathrm{X}$ & $\sqrt{ }$ \\
\hline
\end{tabular}




\section{PREVIOUS TECHNIQUES}

Several studies have been carried out by the investigators in the area of optimization and operation techniques and most of them were conducted in the last twenty years. The evaluation of current procedure that is performed to determine the Patient admission scheduling problem (PAS) by using Bilgin et al. dataset. Another study related to PAS is considered in conformity with comprehensive attention of the administration in hospitals, wherein the intensive treatment unit and day clinics depth care gadgets and day clinics are discussed by Bilgin et al. ${ }^{12}$ The another finding of the late acceptance hill climbing algorithm is also implemented on the exam timetabling problems (Burke and Bykov. ${ }^{12}$ Further material about the exam timetabling studies may be determined in a number of survey papers as well as Carter et al. ${ }^{13}$, Schaerf ${ }^{14}$, Burke and Petrovic. ${ }^{15}$ Furthermore, the recommendation of new patient admission procedure that integrated each static and vigorous environment has also been studied. The authors of their accession thought about the compulsion of stay period and the chances to delay the patients' admission at the same time as the hazard of congestions are studied. The demonstration of a pattern for fixing the PAS challenges that includes of assets, branch, affected person and their mechanism is investigated by Vermeulen et al. ${ }^{16}$ Some of the investigators have recommended their own transformation of our procedure, for instance: Late Acceptance Randomized Descent algorithmic rogram ${ }^{17}$ or Multi objective Late Acceptance algorithmic program. ${ }^{18}$ Additionally, this methodology was hybridized with different techniques 19 and explored in respect of the recently developed hyper-heuristic approach. ${ }^{20}$ LAHC appears to be as swiftly as easy hill climbing but more influential. This is due to the fact that LAHC is capable to obtain a worse answer primarily accordance with intelligent use of an adaptive memory to maintain data from previous iterations and reprocess system. ${ }^{21}$

\section{FUNDAMENTAL OF HILL CLIMBING SOLUTION}

An iterative indigenous searching technique of Late Acceptance Hill Climbing (LAHC) specifically enlivened by simple hill climbing algorithm. It is another heuristic technique that used for numerous approaches in getting away from local search. The strategy of LAHC begins from the underlying solution and iteratively enhances the specific solution in respect to produce another ideal one. Fundamentally, the oldstyle slope climbing analyzer is avaricious and quick in nature, it could be effectively and rapidly point out in a neighborhood. The motivation to improve the demonstration of the traditional slope climbing stimulate the presentation of LAHC. The LAHC review the examination of the arrangement by recollecting the previous information and addition of current arrangements in a rundown of a specific length, called wellness cost cluster $f(x)$ of length $L f(x)$ where $\quad(f(x)=$ $\{f(0), f(1), \ldots, f(L f(x-1))\})$. Note $\mathrm{f}(\mathrm{x})$ is the wellness cost estimation of arrangement $\mathrm{x}$ and when $\mathrm{L} \mathrm{f}(\mathrm{x})=1$, at that point the LAHC comes back to the traditional slope climbing. In LAHC, an influential arrangement isn't contrasts and its previous and current component has managed by cost cluster. In this way the streamlining agent acknowledge a degenerating competitor arrangement. Their wellness cost is superior to anything the one formed prior to 1 cycles. The procedure helps the LAHC in getting away nearby ideal as on account of a traditional slope climbing. It is qualified to make reference to all components of the rundown and contributed similar qualities such as equivalent to the wellness cost. Algorithm 1 displays the pseudocode of LAHC system.

\begin{tabular}{|c|c|}
\hline \multicolumn{2}{|c|}{ Algorithm 1 Pseudocode of LAHC procedure } \\
\hline 1: & Generate an initial solution $\boldsymbol{w}$ \\
\hline $2:$ & Calculate initial fitness cost $f(w)$ \\
\hline 3: & Set initial number of steps $\boldsymbol{l}=\mathbf{0}$ \\
\hline 4: & for $k \in(0 \ldots . . l-1) f(k)=f(w) d o$ \\
\hline 5: & repeat \\
\hline $6:$ & Construct a patients solution $w^{\prime}$ \\
\hline 7: & Calculate its cost function $f\left(w^{\prime}\right)$ \\
\hline 8: & $g=I \bmod l$ \\
\hline 9: & if $f\left(w^{\prime}\right) \leq f(g)$ then \\
\hline 10: & Then accept $w^{\prime}$ \\
\hline 11: & end if \\
\hline 12: & Insert cost value into list $f(g)=f(w)$ \\
\hline 13: & Increment a number of steps $l=l+1$ \\
\hline 14: & until (termination criterion are met) \\
\hline 15: & end for \\
\hline
\end{tabular}

\section{PROPOSED LAHC - BASED PFP ALGORITHM AND EXPERIEMNTAL SETUP}

The adjustment of LAHC-based PFP, where patient facing problems in hospitals. The parameter of the LAHC-based PFP like the Maxcycle, that is related to several iteration and express the number of paramedical staff administered (or object cost array) used for the PFP are modified. In the same way PFP are generally derived from the problem examples. These expressions incorporate the arrangement of the 
set of doctors and nurse's communication with patients (see Table 1). Patient is the fundamental outcome variable of the study. Every patient might be treated well and get quick response from the paramedical staff. The pesudocode of the LAHC-based PFP is assumed in Algorithm.

To evaluate the effectiveness of the Late Acceptance Hill Climbing (LAHC) method in the evaluation of patient's satisfaction regarding hospital performance, we have conducted experiment on the datasets of Hospital consumer assessment of healthcare providers and systems (HCAHPS). Total 2009 patients interviewed in nine different public hospitals. The program of the study has coded on Java NetBean version 6.0 and datasets can be accessed from [https://data.cityofnewyork.us/Health/NYC-HealthHospitals-patient-satisfaction-scores-2/hi3x-y76v].

TABLE I listed out the characteristics of each dataset.

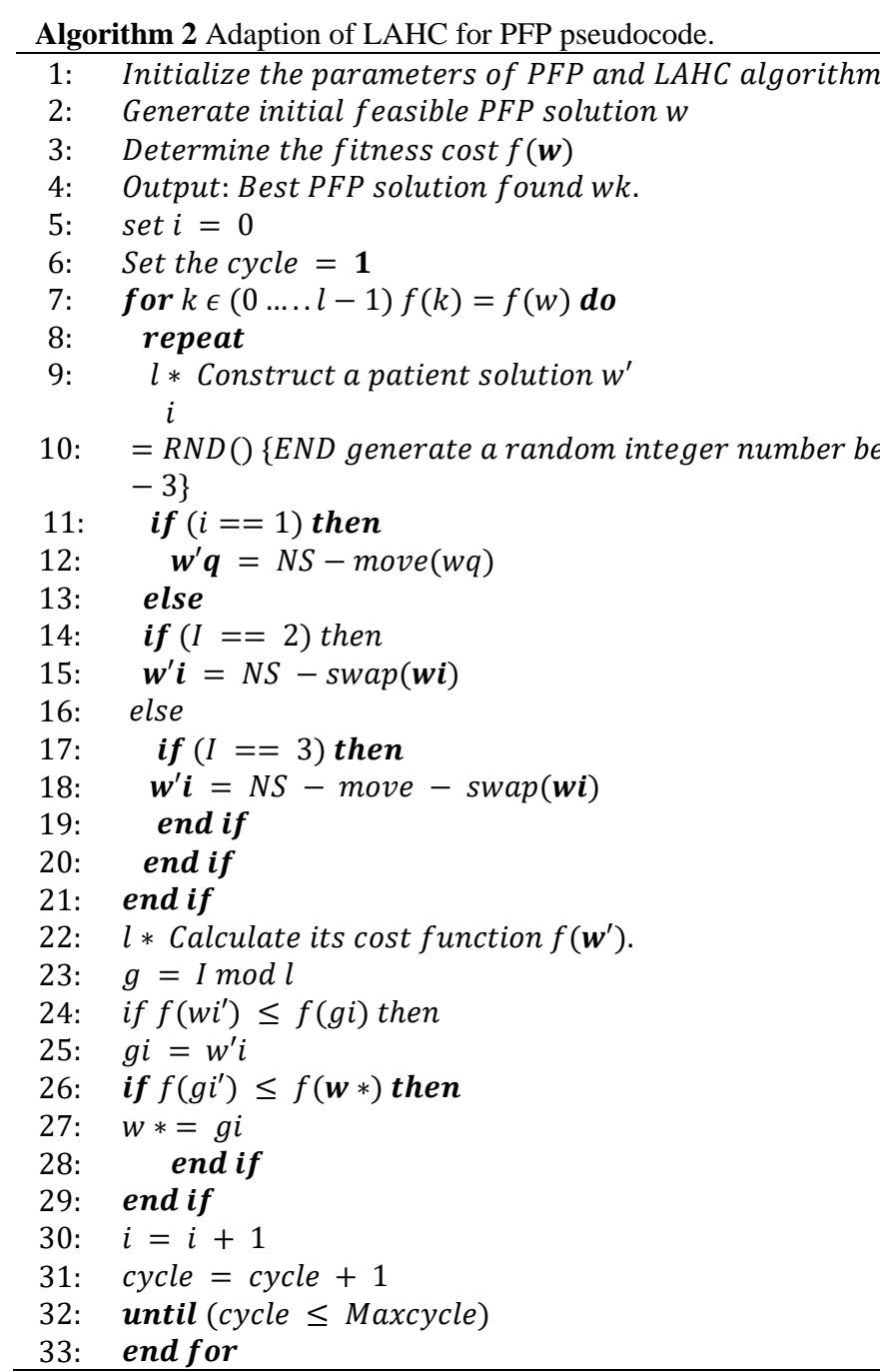

\section{RESULTS}

The analysis has conducted on six common statements of PFP data set. The mean and standard deviation of the scores of doctors and nurses communication well with patients is $70.55 \pm 6.864$ and response time to attend the patients from the paramedical staff is 49.45 \pm 4.083 . After receiving the treatment patient get early symptomatic relief score is $57.64 \pm 3.042$. Clean hospital environment particularly rooms and bathrooms score is $63.36 \pm 4.717$. Majority of the patients agreed that doctor gives instructions to what to do during their recovery at home, so the score is 78.55 \pm 2.423 . (Table 2). In the same way, the results observed in case 3 displayed the version of LAHC algorithm. Although, inserted entity relocates and interchange which is able compete with case 1 and indicate that the incorporation of these neighborhood searches within the component of the LAHC could be explored navigation of the algorithm. and therefore, enhances the efficiency. Moreover, the case 2 along entity relocate and entity swap neighborhoods is closer to that case 3 with respect to the penalty cost is approximately same in accordance with the persisting cases 3, 5, 2 and 6. Besides, the results obtained by the LAHC algorithm is strengthened by reducing the penalty cost further with changing the insertion of these neighborhoods (i.e. Case 5 and 6). Hence, it very well may be seen that the outcomes acquired demonstrate that consolidation of at least two tneighblorhoods with the administrators of LAHC calculation improves the search ability of the recommended algorithm.

\section{CONCLUSION}

There is large number of patients visiting hospitals due to different conditions. The resources are limited In healthcare institutions. Hospital management attempts to meet patient's satisfaction by considering their preferences about the healthcare services. Our study, propose late acceptance of hill climbing for solving six most common problems faced by patients in hospitals using standard dataset. The recommended algorithm is modified to deal with the optimization nature of the PFP and also provide feasible explanation to obtain the solution. We thoroughly examined different variables of patient's satisfaction and applied LAHC by using three neighborhood structures. This mechanism makes LAHC-based PFP more competent and appropriate when investigated to manage various problems. 


\section{REFERENCES:}

1. Park JE. Man and medicine: towards health for all. In: Bhanot B, Preventive and social medicine, $22 \mathrm{nd}$ ed. Jabalpur: 2002, pp. 1-11.

2. W. Vancroonenburg, D. Goossens, F. Spieksma, on the complexity of the patient assignment problem, Techical report, KAHO Sint-Lieven, Gebroed- ers De Smetstraat 1, Gent, Belgium, 2011. http://allserv.kahosl.be/wimvc/ pas- complexitytechreport.pdf

3. B. Bilgin, P. Demeester, G. Vanden Berghe, $A$ hyperheuristic approach to the patient admission scheduling problem, Techical Report, KaHo SintLieven, Gent (2008) .

4. McCollum, B.: A perspective on bridging the gap between theory and practice in university timetabling. In: Burke, E.K., Rudov'a, H. (eds.) PATAT 2006. LNCS, vol. 3867, pp. 3-23. Springer, Heidelberg (2007). https://doi.org/10.1007/978-3- 540-77345-0 1

5. "Ulker, " O., Landa-Silva, D.: A 0/1 integer programming model for the office space allocation problem. Electron. Not. Discrete Math. 36, 575-582 (2010) Adaptation of LAHC Algorithm for Optimizing the OFA Problem 189

6. Benjamin, C.O., Ehie, I.C., Omurtag, Y.: Planning facilities at the University of Missouri-Rolla. Interfaces 22(4), 95-105 (1992)

7. Yuan, B., Zhang, C., Shao, X.: A late acceptance hill-climbing algorithm for balancing two-sided assembly lines with multiple constraints. J. Intell. Manuf. 26(1), 159-168 (2015)

8. Abuhamdah, A.: Experimental result of late acceptance randomized descent algorithm for solving course timetabling problems. Int. J. Comput. Sci. Netw. Secur.10(1), 192-200 (2010)

9. Verstichel, J., Berghe, G.V.: A late acceptance algorithm for the lock scheduling problem. In: Voß, S., Pahl, J., Schwarze, S. (eds.) Logistik Management, pp. 457- 478. Springer, Heidelberg (2009)

10. Tierney, K.: Late acceptance hill climbing for the liner shipping fleet repositioning problem. In: Proceedings of the 14th EU/MEWorkshop, pp. 21-27 (2013) 190 A. L. Bolaji et al.

11. Goerler, A., Schulte, F., Voß, S.: An application of late acceptance hill-climbing to the traveling purchaser problem. In: Pacino, D., Voß, S., Jensen, R.M. (eds.) ICCL 2013. LNCS, vol. 8197, pp. 173-
183. Springer, Heidelberg (2013). https://doi.org/ 10.1007/978-3-642-41019-2 13

12. B. Bilgin, P. Demeester, G. Vanden Berghe, $A$ hyperheuristic approach to the patient admission scheduling problem, Techical Report, KaHo SintLieven, Gent (2008) .

13. Burke E. K., \& Bykov, Y. (2008). A late acceptance strategy in hillclimbing for exam timetabling problems: Proceedings of the 7th International Conference on the Practice and Theory of Automated Timetabling (PATAT2008), Montreal, Canada 13 Di Gaspero, L.,\&Schaerf, A. (2001). Tabu search techniques for examination timetabling. Practice and Theory of Automated Timetabling III, Lecture Notes in Computer Science. Berlin: Springer.

14. Di Gaspero, L.,\&Schaerf, A. (2001). Tabu search techniques for examination timetabling. Practice and Theory of Automated Timetabling III, Lecture Notes in Computer Science. Berlin: Springer.

15. Bykov Y., \& Petrovic, S. (2013). An initial study of a novel Step Counting Hill Climbing heuristic applied to timetabling problems: Proceedings of 6th Multidisciplinary International Scheduling Conference (MISTA 2013), Gent, Belgium.

16. I. Vermeulen, S. Bohte, K. Somefun , H. La Poutré, Multi-agent Pareto appoint- ment exchanging in hospital patient scheduling, Serv. Oriented Comput.Appl. 1 (3) (2007) 185-196.

17 Abuhamdah, A. (2010). Experimental result of late acceptance randomized descent algorithm for solving course timetabling problems. International Journal of Com- puter Science and Network Security, 10, 192200. Alzaqebah, M. , \& A

18. Vancroonenburg, W. \& Wauters, T. (2013). Extending the late acceptance meta- heuristic for multi-objective optimization. In Proceedings of the 6th multidis- ciplinary international scheduling conference: Theory \& applications (MISTA2013) (pp. 652-655). August 2013.

19. Alzaqebah, M., \& Abdullah, S. (2014). An adaptive artificial bee colony and late acceptance hill-climbing algorithm for examination timetabling. Journal of Scheduling, 17 (3), 249-262

20. Jackson, W., Özcan, E., \& Drake, J. H. (2013). Late Acceptance-based selection hy- per-heuristics for cross-domain heuristic search. In Proceedings of 13th 
annual workshop on computational intelligence (UKCI), September 2013, Guildford, UK (pp. 228235).

21. Burke, E. K., \& Bykov, Y. (2012). The late acceptance hill-climbing heuristic, technical report CSM-192. Stirlimg: Computing Science and Maematics, University of Stirling. 